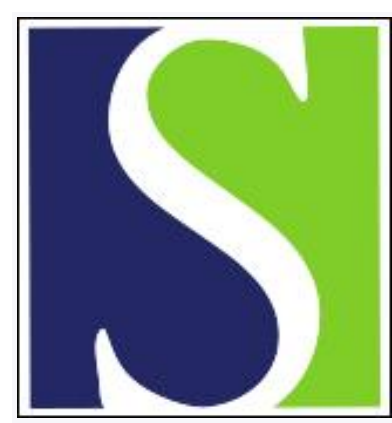

Scand J Work Environ Health 1998;24(4):241-244

https://doi.org/10.5271/sjweh.316

Issue date: Aug 1998

Solving mysteries of the bioeffects of nonionizing radiation

by Hietanen $M$

The following article refers to this text: 2002;28(4):264-269

This article in PubMed: www.ncbi.nlm.nih.gov/pubmed/9754854

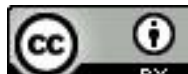


Scand J Work Environ Health 1998;24(4):241-243

\section{Solving mysteries of the bioeffects of nonionizing radiation}

Every summer in Scandinavia, many severely burned people, mostly young girls, need intensive medical care. The reason is not the bonfires lit during the traditional celebration of midsummer; instead it is overexposure to the sun. In spite of the fact that people should be more and more educated concerning the health hazards caused by solar ultraviolet (UV) radiation, all generations seem to need "once bitten, twice shy" experience. Although the painful sunburn will fade gradually after a few days or weeks, there may still be serious late effects. UV exposure has been indicated as the main factor in the induction of nonmelanocytic skin cancers. Besides, epidemiologic studies on cutaneous melanoma suggest that sunburn of the skin may be of particular importance in its initiation.

UV radiation is one form of nonionizing electromagnetic (EM) radiation whose primary mode of interaction with matter is other than ionization. All EM radiation is characterized by frequency $f$ and wavelength $\lambda$ linked together by the relationship $f=c / \lambda$, where $c$ is the speed of light. The nonionizing spectrum contains a large range of wavelengths and frequencies from vacuum UV to static electric and magnetic fields. Biological effects of EM radiation depend greatly on wavelength and various other physical parameters. Consequently, its categories usually basically reflect different biological interactions. Optical radiation consists of UV, visible, and infrared radiation. The other areas of the EM spectrum contain static, extremely low frequency, low frequency, and radiofrequency radiation. Except in the narrow band of visible radiation, called light, EM radiation is invisible to the human eye and unperceived also by other senses at normal environmental levels.

For optical radiation, the sun is globally the most significant source of occupational and environmental exposure. While the intensity of solar radiation depends on the latitude, being the most effective at the equator, it is worthwhile to note some peculiarities concerning solar conditions in Scandinavia. In summer, the daily length of time that the sun shines is long when compared, for example, with the more southern parts of Europe. The height of the sun is predominantly low, so that it is difficult to avoid looking at it. The third contributing factor to UV exposure is the reflection of optical radiation from snow and ice. Hence, outdoor workers, for example, fishermen, merchants at marketplaces, and road builders, are at risk of chronic overexposure to solar UV radiation.

As for the adverse effects of visible radiation on the eye, there are numerous epidemiologic studies indicating that chronic exposure to shortwave light, also called blue light, is a contributing factor to the aging and degeneration of the retina. Several studies have revealed that the shorter the wavelength, the more severe the ocular photochemical damage. Fortunately, the human eye is protected by an aversion reflex against hazardous light sources. Therefore, we cannot look at the sun when it is high and bright and, hence, is dangerous for the retina. Even though the daily solar spectrum changes with the height of the sun, comfortable viewing of it is possible only at a Zenith angle of less than a few degrees - a moment after the sunrise or before the sunset.

Although the eyelids normally close at exposure to sudden bright light, this aversion response is too slow in special situations. Especially hazardous are laser beams, which can focus on a small spot on the retina and result in permanent visual impairment. During the last couple of years, small but potentially hazardous laser pointers have been rushed to the market and are used as popular toys among children. Given the equivocal understanding of laser radiation by the general public, specific restrictions are under consideration in some countries to ensure safe use of these devices. 
The aforementioned examples indicate that the health risks of high exposure to the optical region of the EM spectrum are incontestable and well established. Albeit adverse effects of overexposure to UV and visible radiation are generally well-known, enlightenment campaigns emphasizing the importance of individual responsibility and the necessity of personal protection should be part of occupational and environmental health education.

As with optical radiation, people in technically developed countries are surrounded by radiofrequency radiation. Since field levels of natural background sources are several orders lower than those emitted by human-made sources, exposure is practically always caused by sources that emit EM fields either intentionally or as by-products. Whole-body or localized exposure to radiofrequency fields induces body currents and energy absorption in tissues, depending on the specific frequencies and coupling mechanisms. Thermal effects caused by temperature rises are obvious and basically understood, whereas the principal scientific challenge is to understand the suspected or hypothesized nonthermal effects.

At the moment, radiofrequency fields around the frequencies of $900 \mathrm{MHz}$ and $1800 \mathrm{MHz}$ are of special interest because of the rapid advances in telecommunication technology. As a result of the expansion of the use of handheld cellular phones, the focus of research efforts has moved from power lines to radiotelephone handsets and base station antennas. This issue of the Scandinavian Journal of Work, Environment \& Health provides a review article on biological research on amplitude-modulated radiofrequency radiation, emitted, for example, by digital GSM phones. Apparently the levels of these fields are so low that the mechanism of temperature rise is unlikely to explain the alleged adverse health effects. Other mechanisms of interaction have been proposed, but biological experiments have failed to confirm their existence. Finally, a new mystery has arisen as some occupationally or nonoccupationally exposed persons in some countries have begun to claim specific hypersensitive reactions during or after exposure to radiofrequency fields.

Several international bodies have realized the necessity of expanding interest in radiofrequency radiation research in order to identify gaps in scientific knowledge. For example, the World Health Organization (WHO) has launched the 5-year International EMF Project for providing independent scientific assessments of health effects from exposure to EM fields. The project will encourage the setting of internationally accessible guidelines and standards for exposure limits and device emissions. It will also provide information on the risk perception, risk communication, and risk management of EM fields. In addition, it will provide guidance for scientific research programs. The principal scientific organization supporting the work by WHO on nonionizing radiation is the International Commission on Non-lonizing Radiation Protection (ICNIRP). The main activity of ICNIRP is to provide guidance on safe exposure and protection for workers and members of the public by issuing statements and recommendations. The members of ICNIRP are selected on the basis of their independence and expertise. In order to assist with the process of reviewing research results, four standing committees, consisting of ICNIRP members and external specialists, have been formed for various EM subregions. Further information on WHO and ICNIRP activities in the area of EM radiation can be found at the following Internet addresses: http://www.who.ch/emf/ and http://www.icnirp.de.

A great deal of scientific interest in investigating the health effects of extremely low-frequency and radiofrequency radiation exists also in Europe, within the EU Framework Programmes of Science, Research and Development. Recently, the European Commission formed an expert group to make recommendations for a research program for health hazard assessments of the use of cellular radiotelephones. A group of scientists invited by the Directorate General is drafting a proposal for a council recommendation for limiting public exposure to EM radiation. Moreover, scientific collaboration within research on the biological effects of EM fields was started already several years ago as a part of COST (European Co-operation in the Field of Scientific and Technical Research). 
As for future research strategies on EM radiation, the modern international scientific community offers good opportunities for an interdisciplinary approach of high standard. Since EM fields are ubiquitous in our work and living environments, it is important that investigations on possible health effects caused by low exposure levels not be neglected, in spite of the hard work involved with probably slow progress. This need for knowledge imposes stringent requirements for research laboratories and requires a great deal of material support and funding by different sources, including industry manufacturing EM radiation sources. Encouragement of funding agencies to support this research will ultimately help in creating a reliable scientific data base on which health risk assessments can be founded.

Maila Hietanen, $\mathrm{PhD}$

Finnish Institute of Occupational Health

Laajaniityntie 1

FIN-01620 Vantaa Finland

e-mail: Maila.Hietanen@occuphealth.fi 